\title{
芳香重氮盐参与的协同环加成反应
}

\author{
彭星, 张发光 ${ }^{*}$, 马军安 ${ }^{*}$ \\ 天津大学理学院化学系, 天津 300072
}

\begin{abstract}
摘要: 芳香重氮盐由于合成简单及多重反应性, 在有机合成中占有重要地位。尽管芳香重氮盐的脱氮气交叉偶联反 应、还原反应及与富电子芳烃的偶合反应已得到广泛应用, 但是其参与的环加成反应却长期未受重视, 在大学化学 学习中也无相关介绍。值得注意的是, 近年芳香重氮盐参与的成环反应日益增多, 也为丰富和发展多样性的含氮杂 环化合物提供了一种有效途径。因此, 本文概述了芳香重氮盐协同环加成反应的最新进展及其在杂环合成方面的全 新应用和机遇。
\end{abstract}

关键词: 芳香重氮盐; 环加成反应; 四氮唑; 三氮唑

中图分类号: G64; O6

\section{Aryl Diazonium Salts in Concerted Cycloaddition Reactions}

\section{Xing Peng, Fa-Guang Zhang *, Jun-An Ma *}

Department of Chemistry, School of Science, Tianjin University, Tianjin 300072, China.

\begin{abstract}
Aryl diazonium salts are a class of important synthons in the field of organic synthesis due to their versatile reactivity and easy availability. Despite the widespread use of the denitrogenation-type cross-coupling, reduction, and azo-coupling reactions of aryl diazonium salts, the cycloaddition reactions of aryl diazonium salts have long been neglected. Noteworthy is that recent renaissance in this type of reactions has open powerful access to a diverse array of $\mathrm{N}$-heterocycles. This minireview summarizes the latest development and applications of cycloaddition reactions of aryl diazonium salts as well as their new opportunity in organic synthesis.
\end{abstract}

Key Words: Aryl diazonium salts; Cycloaddition; Tetrazoles; Triazoles

芳香重氮盐是有机合成领域一类非常重要的中间体, 在有机化学、药物化学以及材料科学等领 域具有广泛的应用价值 ${ }^{[1]}$ 。尽管芳香重氮盐的发现可以追溯到 1858 年 ${ }^{[2]}$, 但在相当长的一段时期内它 鲜被关注, 这主要是因为最初报道的芳基重氮盐采用氯离子作为平衡阴离子, 而此类芳香重氮盐在 零度以上极度不稳定且具有爆炸性。非常幸运的是, 后期研究发现芳香重氮盐可以通过调整平衡阴 离子的种类来提高稳定性, 已经报道的具有良好稳定性的平衡阴离子包括四氟嗍酸阴离子以及六氟 磷酸阴离子等 ${ }^{[3]}$ 。此后, 芳香重氮盐在多个领域受到人们广泛关注, 主要是因为以下几点: 1) 原料 芳香胺廉价易得, 便于大量制备; 2) 反应条件温和, 多在室温下进行; 3) 反应类型多样; 4) 化学 选择性较高。

芳香重氮盐参与的化学反应主要可分为两大类(图1 $\left.{ }^{[4]}: 1\right)$ 脱除双氮反应, 包括自由基或碳正离

收稿: 2021-01-13; 录用: 2021-03-03; 网络发表: 2021-03-29

“通讯作者, Emails: zhangfg1987@tju.edu.cn (张发光); majun_an68@tju.edu.cn (马军安)

基金资助：国家自然科学基金(21772142，21901181，21961142015) 
子历程的交叉偶联反应, 如Sandmeyer反应 ${ }^{[5]}$ 、Gomberg-Bachmann反应 ${ }^{[6]}$ 、Balz-Schiemann反应 ${ }^{[7]}$ 、 Meerwein芳基化 ${ }^{[8]}$ 等人名反应(图1A); 近年来, 芳香重氮盐在金属催化的偶联反应(如Heck-Matsuda 反应)及碳氢键活化等领域也得到大量应用 ${ }^{[9]}$ 。此类化学转化可用于合成多种卤代芳烃、芳香腈、取 代苯酚以及联芳基化合物等; 2) 保留双氮反应, 主要为重氮盐 $\mathrm{N} \equiv \mathrm{N}$ 键的直接还原及其与富电子芳烃 的偶合反应, 该类官能团转化可用于合成芳基肼和偶氮类化合物(图 $1 \mathrm{~B})^{[10]}$ 。上述两类化学转化已在 有机合成等领域得到广泛应用, 在大学化学中也被重点介绍, 属于芳香重氮盐的经典反应类型 ${ }^{[11]}$ 。

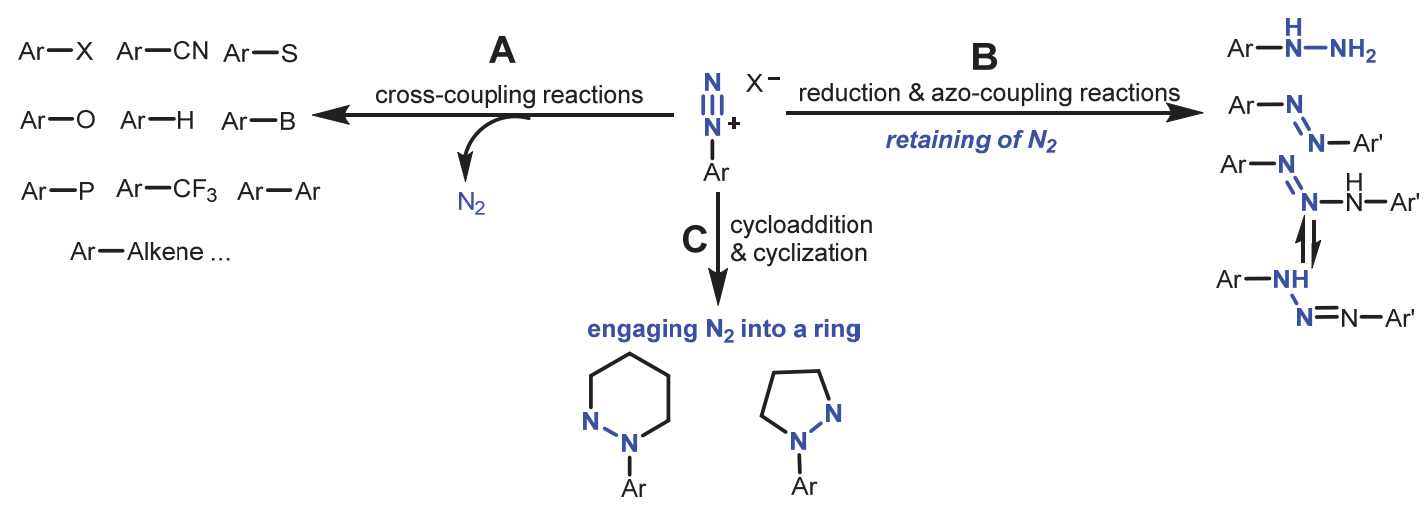

图1 芳香重氮盐参与的化学反应

值得注意的是, 近年来芳香重氮盐参与的环加成及环化反应也相继被报道, 该类反应在保留两 个氮原子的同时, 可以成功将芳香重氮盐转化为含氮杂环化合物, 具有良好的原子经济性及合成应 用价值(图1C) ${ }^{[12]}$ 。其中, 芳香重氮盐参与的协同环加成反应是此类成环反应中最具挑战性的研究课 题, 如何兼顾重氮盐稳定性、反应活性及反应选择性是需要重点考虑的科学问题, 也是值得广大科 研教学工作者及学生们关注的研究方向。因此, 本文主要根据反应类型及产物对芳香重氮盐参与的 协同环加成反应进行分类概括, 旨在分析和总结近年来芳香重氮盐参与的环加成反应进展以及面临 的挑战, 为进一步丰富和发展该类反应提供参考。

\section{1 芳香重氮盐参与的 $[4+2]$ 协同环加成反应}

Diels-Alder反应是经典的构建六元环状化合物的高效方法, 并于 1950 年获得诺贝尔化学奖, 其 中典型的亲双烯体(dienophile)主要为含有吸电子取代基的烯烃或炔烃化合物, 而芳香重氮盐作为亲 双烯体的研究相对较少。1919年Meyer ${ }^{[13]}$ 报道了对硝基苯基重氮盐可以同反式-戊二烯发生反应, 但 受限于当时简陃的科研条件, 反应产物被推定为线性结构, 而非环加成产物。直到五十多年后的 1973 年, Sheppard课题组证明了 1,6-二氢哒嗪才是上述反应的真正产物(图2) ${ }^{[14]}$ 。实验表明: 二烯烃的结 构对该反应具有很大影响, 反式-戊二烯能够顺利发生该反应, 而顺式-戊二烯即使反应6小时也观察 不到任何产物的生成, 基于此作者指出该反应经历 $[4+2]$ 协同环加成反应机理; 同时 Sheppard和其合 作者对上述反应进行底物扩展, 发现非硝基取代的芳基重氮盐形成的 1,6-二氢哒嗪产物, 可以进一步 发生原位氧化从而得到哒嗪盐类化合物 ${ }^{[15]}$ 。之后到了1984年, Huisgen及其合作者 ${ }^{[16] 又}$ 研究了对硝基 苯基重氮盐与其他双烯体的 $[4+2]$ 协同环加成反应, 从而得到了一系列高度官能化的 1,6 -哒嗪衍生 物。

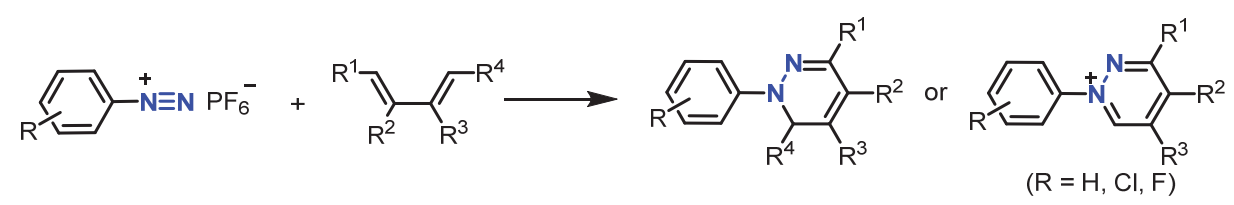

图2 芳香重氮盐与双烯体的 $[4+2]$ 协同环加成反应构建哒嗪及哒嗪盐 


\section{2 芳香重氮盐参与的 $[3+2]$ 偶极环加成反应}

\section{1 四氮唑的合成}

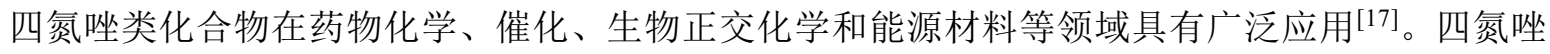

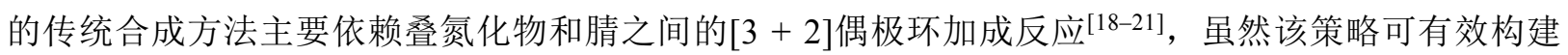
单取代或1,5-双取代四氮唑, 但2,5-双取代四氮唑化合物的合成仍存在着相当大的挑战。1955年德国 Huisgen等人 ${ }^{[22]}$ 发现对硝基苯基重氮盐与重氮甲烷可发生 $[3+2]$ 偶极环加成反应, 以 $12 \%$ 的收率得到 一个芳基四氮唑产物(图3A)。但由于文献没有提供具体实验操作, 重氮甲烷危险性高, 产物收率极 低, 产物结构表征不完备, 并未引起广泛关注。为克服这一局限, 天津大学马军安团队 ${ }^{[23]} 2015$ 年 首次报道了银催化芳香重氮盐与三氟重氮乙烷 $\left(\mathrm{CF}_{3} \mathrm{CHN}_{2}\right)$ 及重氮乙酸酯的 $[3+2]$ 偶极环加成反应 (图3B)。与Huisgen的最初工作相比, 通过使用金属银盐作为催化剂, 该反应的收率、区域选择性及 底物适用范围均得到大幅提升, 从而快速构建了系列含有三氟甲基及酯基的四氮唑类化合物。值得 注意的是, 该反应还可进行重氮化/环加成的一锅操作, 从而实现了从芳香胺到四氮唑的直接转化, 进一步证明了该方法的实用性。

在此基础上, 马军安课题组相继成功实现了银催化下芳香重氮盐与苯磺酰基二氟重氮乙烷

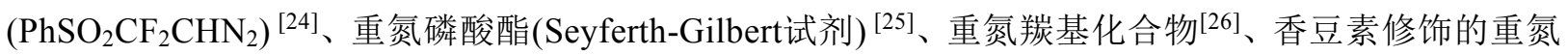
化合物 ${ }^{[27]}$ 以及重氮乙腈 ${ }^{[28]}$ 的 $[3+2]$ 环加成反应(图3C-G)。在温和条件下, 该类反应可分别高效构建 出一系列 5-二氟甲基、5-磷酸酯基、5-羰基、5-单氟甲基、5-香豆素骨架、5-氧基取代的四氮唑类衍 生物。特别指出的是, 在研究芳香重氮盐与重氮乙腈反应时, 在不使用银催化剂的情况下, 还可以 中等至较好的收率得到 1,5 -双取代的产物, 极大丰富和发展了芳香重氮盐的环加成反应范围与四氮 唑类化合物的分子多样性, 并运用理论计算方法对反应机理进行了阐释。鉴于该方法的广泛适用性 和实用性, 此类反应将在四氮唑化学中得到进一步的应用。

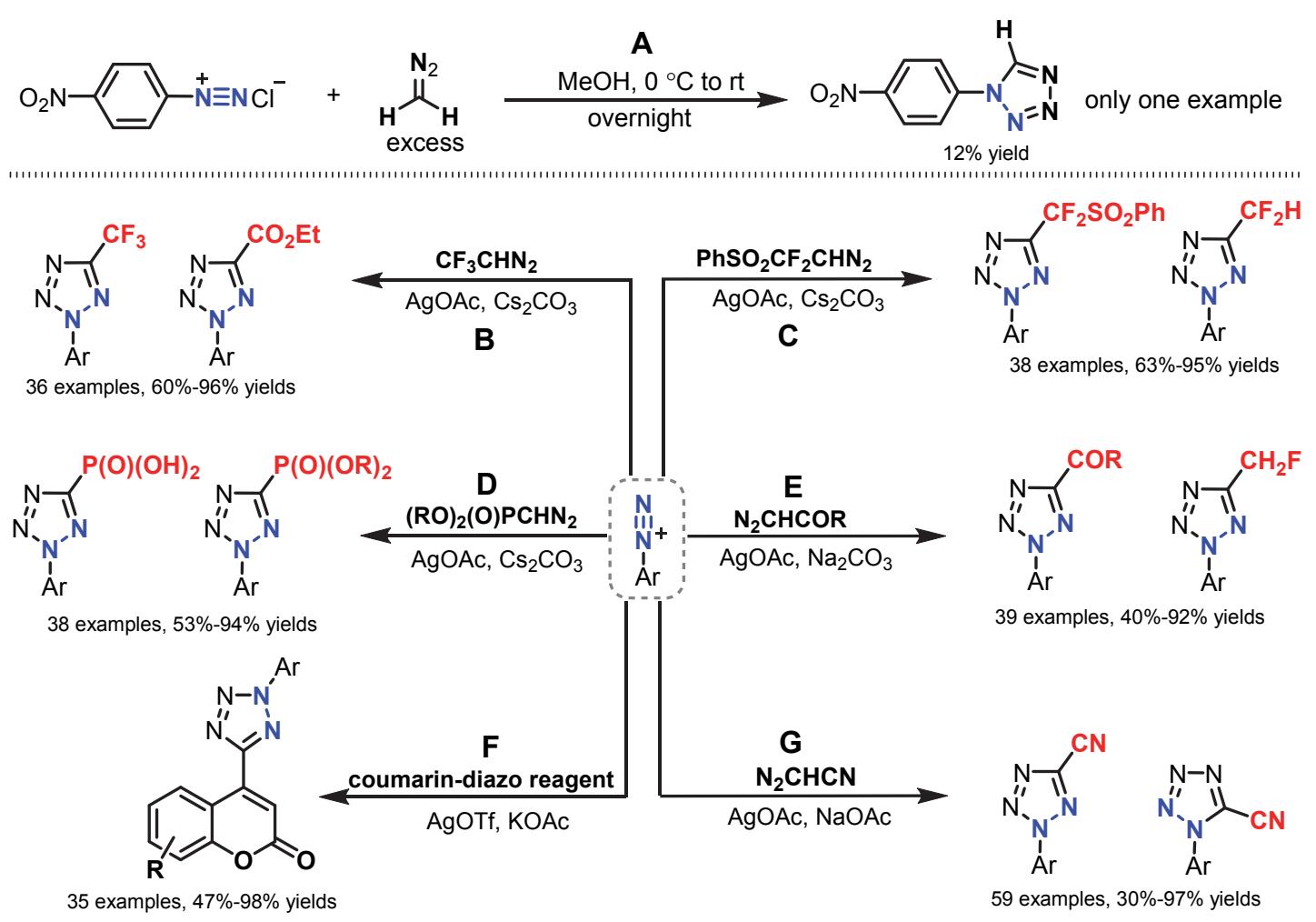

图3 芳香重氮盐与重氮化合物的 $[3+2]$ 偶极环加成反应构建四氮唑类化合物 


\section{2 三氮唑的合成}

三氮唑是药物化学以及材料科学领域的重要结构单元, 主要包括 $1,2,4-$ 三氮唑衍生物和 $1,2,3-$ 三 氮唑衍生物 ${ }^{[29]}$ 。三氮唑的 $\mathrm{N}-\mathrm{H}$ 芳基化或 $\mathrm{C}-\mathrm{H}$ 官能化是获得多取代三氮唑类化合物的常规策略之一, 但是苛刻的反应条件、区域选择性问题和比较局限的底物范围限制了三氮唑类化合物的多样性和功 能性。近年来, 国内学者发展了芳香重氮盐参与的偶极环加成反应, 逐渐成为构建多取代三氮唑类 化合物的一种有效策略。

2018年苏州大学万小兵课题组 ${ }^{[30]}$ 报道了重氮乙酸乙酯、芳香重氮盐和腈的一锅三组分反应, 用 于合成三取代1,2,4-三氮唑(图4A)。机理研究表明, 铜催化剂首先与重氮乙酸乙酯反应形成铜卡宾物 种, 其进一步与腈反应生成腈叶立德中间体, 该 1,3-偶极子与芳基重氮盐的 $[3+2]$ 环加成过程为反应 的关键步骤, 所得三氮唑中间体在碱作用下发生异构, 生成最终的三取代1,2,4-三氮唑产物。随后, 天津大学马军安课题组 ${ }^{[31,32]}$ 进一步实现了芳香重氮盐与三氟重氮乙烷、苯磺酰基二氟重氮乙烷、重 氮乙腈在腈类溶剂中的一锅三组分环加成反应(图4B-D), 可分别用于合成含有三氟甲基、二氟甲基、 氧基取代的1,2,4-三氮唑, 并可应用于药物类似物及手性配体的构建, 有良好的拓展应用潜力。近期, 华中师范大学陈加荣小组 ${ }^{[33]}$ 进一步实现了芳香重氮盐与噁唑啉酮的脱羧 $[3+2]$ 环加成反应(图 $4 \mathbf{E}$ )。 该方法以廉价易得的四丁基氯化铵为催化剂, 噁唑啉酮可原位异构化为甲亚胺叶立德中间体, 该1,3偶极子与芳基重氮盐发生区域选择性 $[3+2]$ 环加成反应, 随后脱除二氧化碳可得一系列三取代 $1,2,4-$ 三氮唑产物, 且反应在室温下即可进行。
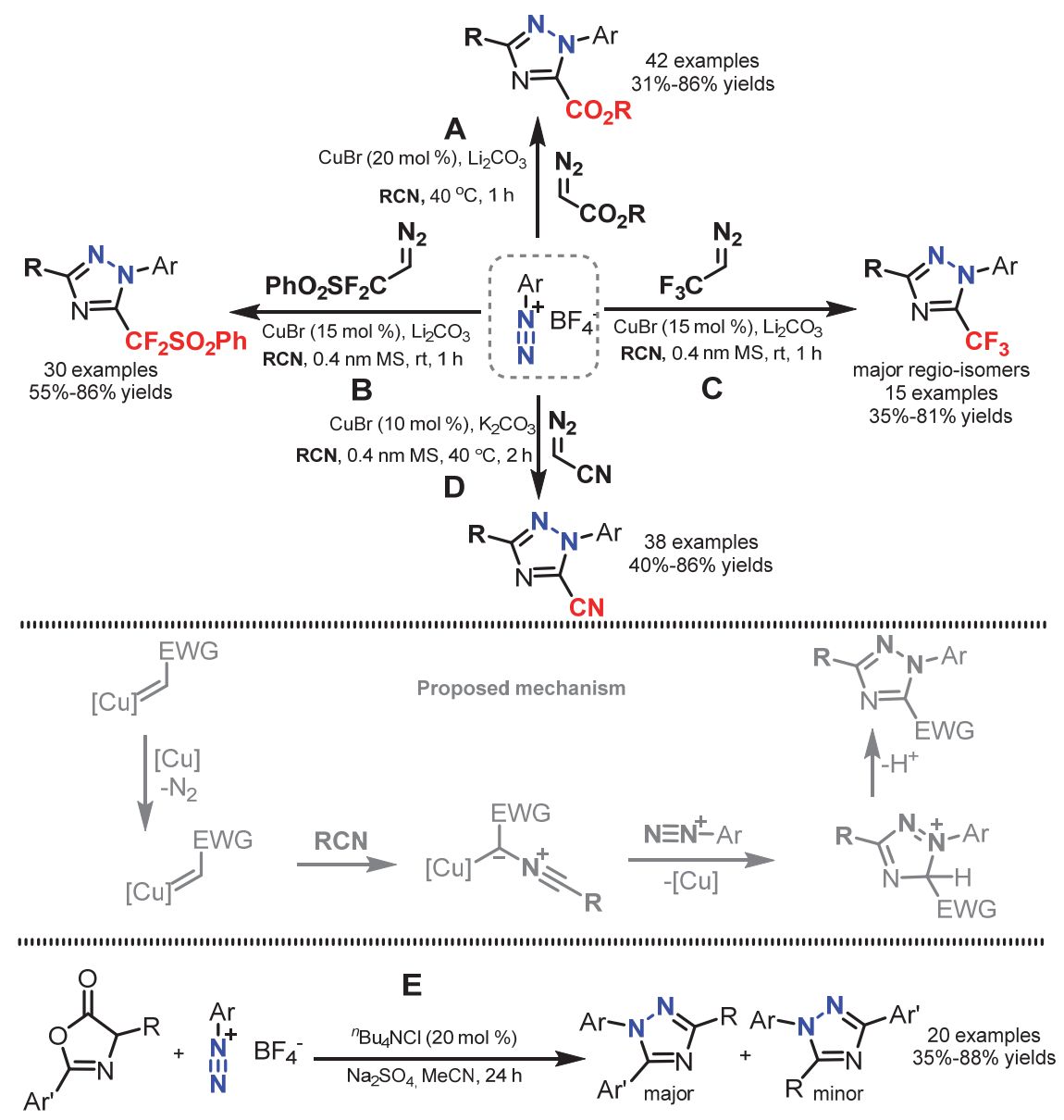

图4 芳香重氮盐与腈叶立德、甲亚胺叶立德的 $[3+2]$ 偶极环加成反应构建三氮唑类化合物 


\section{3 结语}

本文介绍了芳香重氮盐的 $[4+2]$ 与 $[3+2]$ 协同环加成反应, 该类成环转化可有效合成一系列哒 嗪、三氮唑以及四氮唑等杂环化合物。尽管如此, 芳香重氮盐的环加成反应有待进一步发展, 如扩 展可参与反应的双烯体或偶极子类型, 以期合成更加多样的含氮杂环化合物; 相关成环过程的反应 机理仍缺乏深入和系统的研究; 将该类反应应用到生物分子和天然产物的全合成中等。考虑到芳香 重氮盐环加成反应的良好应用前景, 希望本文可以为相关科研教学工作者提供一定的依据和参考。

\section{参 考 文 献}

[1] Gall, C. Chem. Rev. 1988, 88, 765.

[2] Griess, J. P. Justus Liebigs Ann. Chem. 1858, 106, 123.

[3] Oger, N.; d'Halluin, M.; Le Grognec, E.; Felpin, F.-X. Org. Process Res. Dev. 2014, 18, 1786.

[4] Mo, F.; Dong, G.; Zhang, Y.; Wang, J. Org. Biomol. Chem. 2013, 11, 1582.

[5] Mo, F.; Qiu, D.; Zhang, Y.; Wang, J. Acc. Chem. Res. 2018, 51, 496.

[6] Gomberg, M.; Bachmann, W. E. J. Am. Chem. Soc. 1924, 46, 2339.

[7] Balz, G.; Schiemann, G. Ber. Dtsch. Chem. Ges. 1927, 60, 1186.

[8] Hari, D. P.; König, B. Angew. Chem. Int. Ed. 2013, 52, 4734.

[9] Roglans, A.; PlaQuintana, A.; Moreno-Mañas, M. Chem. Rev. 2006, 106, 4622.

[10] Merino, E. Chem. Soc. Rev. 2011, 40, 3835.

[11] 邢其毅, 裴伟伟, 徐瑞秋, 裴坚. 基础有机化学. 第4版. 北京: 北京大学出版社, 2016.

[12] Zhang, F.-G.; Chen, Z.; Cheung, C.-W.; Ma, J.-A. Chin. J. Chem. 2020, 38, 1132.

[13] Meyer, K. H. Ber. Dtsch. Ges. 1919, 52, 1468.

[14] Sheppard, W. A.; Webster, O. W. J. Am. Chem. Soc. 1973, 95, 2695.

[15] Carlson, B. A.; Sheppard, W. A.; Webster, O. W. J. Am. Chem. Soc. 1975, 97, 5291.

[16] Bronberger, F.; Huisgen, R. Tetrahedron Lett. 1984, 25, 57.

[17] Neochoritis, C. G.; Zhao, T.; Dömling, A. Chem. Rev. 2019, 119, 1970.

[18] Demko, Z. P.; Sharpless, K. B. Angew. Chem. Int. Ed. 2002, 41, 2110.

[19] Huisgen, R. Angew. Chem. Int. Ed. 1963, 2, 565.

[20] Breugst, M.; Reissig, H.-U. Angew. Chem. Int. Ed. 2020, 59, 12293.

[21] Liang, L.; Astruc, D. Coord. Chem. Rev. 2011, 255, 2933.

[22] Huisgen, R.; Koch, H.-J. Liebigs Ann. Chem. 1955, 591, 200.

[23] Chen, Z.; Fan, S.-Q.; Zheng, Y.; Ma, J.-A. Chem. Commun. 2015, 51, 16545.

[24] Peng, X.; Xiao, M.-Y.; Zeng, J.-L.; Zhang, F.-G.; Ma, J.-A. Org. Lett. 2019, 21, 4808.

[25] Zhai, S.-J.; Peng, X.; Zhang, F.-G.; Ma, J.-A. Org. Lett. 2019, $21,9884$.

[26] Xiao, M.-Y.; Chen, Z.; Zhang, F.-G.; Ma, J.-A. Tetrahedron 2020, 76, 131063.

[27] Liu, X.-Y.; Zhai, S.-J.; Feng, F.-F.; Zhang, F.-G.; Ma, J.-A. ChemCatChem 2020, 12, 5623.

[28] Xiao, M.-Y.; Zheng, M.-M.; Peng, X.; Xue, X.-S.; Zhang, F.-G.; Ma, J.-A. Org. Lett. 2020, 22, 7762.

[29] Zhang, F.-G.; Peng, X.; Ma, J.-A. Chin. J. Org. Chem. 2019, 39, 109.

[30] Li, H.; Wu, X.; Hao, W.; Li, H.; Zhao, Y.; Wang, Y.; Lian, P.; Zheng, Y.; Bao, X.; Wan, X. Org. Lett. 2018, $20,5224$.

[31] Peng, X.; Zhang, F.-G.; Ma, J.-A. Adv. Synth. Catal. 2020, 362, 4432.

[32] Zhou, L.-N.; Feng, F.-F.; Cheung, C.-W.; Ma, J.-A. Org. Lett. 2021, 23, 739.

[33] Yu, X.-Y.; Xiao, W.-J.; Chen, J.-R. Eur. J. Org. Chem. 2019, 6994. 